\title{
Riemannian Space-times of Gödel Type in Five Dimensions
}

\author{
M.J. Rebouças* and A.F.F Teixeira ${ }^{\dagger}$ \\ * $\dagger$ Centro Brasileiro de Pesquisas Físicas \\ Departamento de Relatividade e Partículas \\ Rua Dr. Xavier Sigaud 150 \\ 22290-180 Rio de Janeiro - RJ, Brazil
}

April 28, 2018

\begin{abstract}
The five-dimensional (5D) Riemannian Gödel-type manifolds are examined in light of the equivalence problem techniques, as formulated by Cartan. The necessary and sufficient conditions for local homogeneity of these 5D manifolds are derived. The local equivalence of these homogeneous Riemannian manifolds is studied. It is found that they are characterized by two essential parameters $\mathrm{m}^{2}$ and $\omega$ : identical pairs $\left(\mathrm{m}^{2}, \omega\right)$ correspond to locally equivalent 5D manifolds. An irreducible set of isometrically nonequivalent 5D locally homogeneous Riemannian Gödel-type metrics are exhibited. A classification of these manifolds based on the essential parameters is presented, and the Killing vector fields as well as the corresponding Lie algebra of each class are determined. It is shown that apart from the $\left(m^{2}=4 \omega^{2}, \omega \neq 0\right)$ and $\left(m^{2} \neq 0, \omega=0\right)$ classes the homogeneous Riemannian Gödel-type manifolds admit a seven-parameter maximal group of isometry $\left(G_{7}\right)$. The special class $\left(m^{2}=4 \omega^{2}, \omega \neq 0\right)$ and the degenerated Gödel-type class $\left(m^{2} \neq 0, \omega=0\right)$ are shown to have a $G_{9}$ as maximal group of motion. The breakdown of causality in these classes of homogeneous Gödel-type manifolds are also examined.
\end{abstract}

*INTERNET:REBOUCAS@CAT.CBPF.BR

${ }^{\dagger}$ INTERNET: TEIXEIRA@NOVELL.CAT.CBPF.BR 


\section{Introduction}

Kaluza-Klein-type theories in five and more dimensions has been of notable interest in several contexts. In the framework of gauge theories they have been used in the quest for unification of the fundamental interactions in physics. The idea that the various interactions in nature might be unified by enlarging the dimensionality of the spacetime, has a long and honourable history that goes back to the works of Nordström, Kaluza and Klein [1] - [3]. Its earlier adherents were mainly interested in extending general relativity, but a late increased interest has been apparent in the particle physics community, especially among those investigating supersymmetry.

The possibility that space-time has more than four dimensions has also received much attention regarding its cosmological aspects. Investigation has been focused on attempts to understand, for example, why the universe presently appears to have only four spacetime dimensions, and whether it is a higher dimensional dynamically evolving manifold the space-time expands while the extra dimensions contract or remain constant. The first cosmological model in which the extra dimension contracts as a result of the cosmological evolution was proposed by Chodos and Detweiler [4]. Since then, a great deal of work has emerged along this line of research, particularly as regards exact solutions of Einstein's equations, entropy production during the contracting process [5], resolution of the horizon and flatness problems [6], and the like (see [7] and references therein).

From a purely technical viewpoint higher-dimensional Kaluza-Klein-type theories have also been used as a way of finding new exact solutions of Einstein's equations in four dimensions [8] - [10].

More recently Wesson [1] - [13] has given a new impetus to the study of $(4+1)$-gravity 
by investigating a five-dimensional extension of general relativity with a variable rest mass (see also [14] and [15]). In this theory we have space-time-mass (STM) Riemannian manifolds, and the fifth dimension is a convenient mathematical way of geometrizing the rest mass and of allowing one to study the possibility that it may be variable. The four-dimensional (4D) general relativity theory is recovered when the rate of change of the rest mass is zero. Ever since the foundations of this Kaluza-Klein-type STM theory were laid, there have been investigations on its potentialities and physical consequences, particularly as concerns its consistency with Mach's principle [16], causality conditions and inflation [14, 17], confrontation between theory and observation [18, 19], and the solutions of its field equations [15].

In 1949 Gödel found a solution of Einstein's field equations with cosmological constant for incoherent matter with rotation [20]. Owing to its striking properties, the cosmological solution presented by Gödel has a well-recognized importance and has to a large extent motivated the investigations on rotating cosmological space-times within the framework of general relativity. Particularly, the search for rotating Gödel-type space-times has received a good deal of attention in recent years, and the literature on these geometries is fairly large today see 21] - 34 and references therein).

However, the general problem of space-time local homogeneity (ST homogeneity, hereafter [35]) of four-dimensional Riemannian manifolds endowed with a Gödel-type metric was considered for the first time only in 1980 by Raychaudhuri and Thakurta [36]. They found the necessary conditions for ST homogeneity of these manifolds. Three years later, Rebouças and Tiomno [37] proved that Raychaudhuri-Thakurta necessary conditions are also sufficient for ST homogeneity of Gödel-type manifolds. However, in both articles [36, 37] the study of ST homogeneity is limited in that only time-independent 
Killing vector fields were considered [38]. The Raychaudhuri-Thakurta-Rebouças-Tiomno conditions were finally proved to be the necessary and sufficient conditions for a Gödeltype manifold to be ST homogeneous without assuming any such simplifying hypothesis in [39], where the powerful equivalence problem techniques for Riemannian space-times, as formulated in terms of spinors by Karlhede [40] and embodied in a suite of computer algebra programs called CLASSI [41] written in SHEEP [42], were used.

In the light of the equivalence problem techniques, as formulated by Cartan [43] and using CLASSI, we extend these investigations by examining a class of five-dimensional (5D) Riemannian Gödel-type manifolds. The necessary and sufficient conditions for local homogeneity of these 5D manifolds are derived. The local equivalence of these homogeneous space-time-mass Riemannian manifolds is discussed; they are found to be characterized by two essential parameters $m^{2}$ and $\omega$ : identical pairs $\left(m^{2}, \omega\right)$ correspond to locally equivalent STM manifolds. An irreducible set of isometrically nonequivalent 5D homogeneous Riemannian Gödel-type metrics are exhibited. A classification of these manifolds based on the essential parameters is presented, and the Killing vector fields as well as the corresponding Lie algebra of each class are determined. We show that apart from the $\left(m^{2}=4 \omega^{2}, \omega \neq 0\right)$ and $\left(m^{2} \neq 0, \omega=0\right)$ classes the 5D locally homogeneous Riemannian Gödel-type manifolds have a seven-parameter maximal group of isometry $\left(G_{7}\right)$. The special class $\left(m^{2}=4 \omega^{2}, \omega \neq 0\right)$ and the degenerated Gödel-type class $\left(m^{2} \neq 0, \omega=0\right)$ are shown to admit a $G_{9}$ as maximal group of motion. The breakdown of causality in these classes of homogeneous Gödel-type manifolds is also examined and shown to have the same basic features of the corresponding $4 \mathrm{D}$ counterparts.

To close this introduction, we should like to emphasize that although the STM Wesson's theory of gravitation is often referred to, the results of the following sections hold 
for any five-dimensional Riemannian Gödel-type manifold regardless of the underlying 5D Kaluza-Klein-type theory[44] - [47] one may be concerned with.

\section{Homogeneity, Irreducible Set and Causality}

The arbitrariness in the choice of coordinates in the geometric theories of gravitation gives rise to the problem of deciding whether or not two Riemannian manifolds whose metrics $g$ and $\tilde{g}$ are given explicitly in terms of coordinates, viz.,

$$
d s^{2}=g_{\mu \nu} d x^{\mu} d x^{\nu} \quad \text { and } \quad d \tilde{s}^{2}=\tilde{g}_{\mu \nu} d \tilde{x}^{\mu} d \tilde{x}^{\nu}
$$

are locally the same. This is the so-called equivalence problem (see Cartan 433 for the local equivalence of $n$-dimensional Riemannian manifolds, Karlhede 40] and MacCallum 42, 48, 49 for the special case $n=4$ of general relativity).

The Cartan solution [43] to the equivalence problem for Riemannian manifolds can be reworded as follows. Two $n$-dimensional Lorentzian Riemannian manifolds $M$ and $\widetilde{M}$ are locally equivalent if there exist coordinate and generalized $n$-dimensional Lorentz transformations such that the following algebraic equations relating the frame components of the curvature tensor and their covariant derivatives:

$$
\begin{aligned}
R_{B C D}^{A} & =\widetilde{R}_{B C D}^{A}, \\
R_{B C D ; M_{1}}^{A} & =\widetilde{R}_{B C D ; M_{1}}^{A}, \\
R_{B C D ; M_{1} M_{2}}^{A} & =\widetilde{R}_{B C D ; M_{1} M_{2}}^{A}, \\
& \vdots \\
R_{B C D ; M_{1} \ldots M_{p+1}}^{A} & =\widetilde{R}_{B C D ; M_{1} \ldots M_{p+1}}^{A}
\end{aligned}
$$

are compatible as equations in $\left(x^{\mu}, \xi^{A}\right)$. Here and in what follows we use a semicolon 
to denote covariant derivatives. Note that $x^{\mu}$ are coordinates on the manifold $M$ while $\xi^{A}$ parametrize the group of allowed frame transformations $[n$-dimensional generalized Lorentz group usually denoted [50] by $O(n-1,1)]$. Reciprocally, equations (2.2) imply local equivalence between the $n$-dimensional manifolds $M$ and $\widetilde{M}$.

In practice, a fixed frame is chosen to perform the calculations so that only coordinates appear in the components of the curvature tensor, i.e. there is no explicit dependence on the parameters $\xi^{A}$ of the generalized Lorentz group.

Another important practical point to be considered, once one wishes to test the local equivalence of two Riemannian manifolds, is that before attempting to solve eqs. (2.2) one can extract and compare partial pieces of information at each step of differentiation as, for example, the number $\left\{t_{0}, t_{1}, \ldots, t_{p}\right\}$ of functionally independent functions of the coordinates $x^{\mu}$ contained in the corresponding set

$$
I_{p}=\left\{R_{B C D}^{A}, R_{B C D ; M_{1}}^{A}, R_{B C D ; M_{1} M_{2}}^{A}, \ldots, R_{B C D ; M_{1} M_{2} \ldots M_{p}}^{A}\right\}
$$

and the isotropy subgroup $\left\{H_{0}, H_{1}, \ldots, H_{p}\right\}$ of the symmetry group $G_{r}$ under which the set corresponding $I_{p}$ is invariant. They must be the same for each step $q=0,1, \cdots, p$ if the manifolds are locally equivalent.

In practice it is also important to note that in calculating the curvature and its covariant derivatives, in a chosen frame, one can stop as soon as one reaches a step at which the $p^{\text {th }}$ derivatives (say) are algebraically expressible in terms of the previous ones, and the residual isotropy group (residual frame freedom) at that step is the same isotropy group of the previous step, i.e. $H_{p}=H_{(p-1)}$. In this case further differentiation will not yield any new piece of information. Actually, if $H_{p}=H_{(p-1)}$ and, in a given frame, the $p^{\text {th }}$ derivative is expressible in terms of its predecessors, for any $q>p$ the $q^{\text {th }}$ derivatives can all be 
expressed in terms of the $0^{t h}, 1^{s t}, \cdots,(p-1)^{t h}$ derivatives 43, 51, 42]. As in the worst case we have only one functionally independent function of the coordinates $x^{\mu}$ at each step of the differentiation process, and the generalized Lorentz group has $n(n-1) / 2$ independent parameters, it follows that for five-dimensional Riemannian manifolds $p+1 \leq 15$.

Since there are $t_{p}$ essential coordinates, in $5 \mathrm{D}$ clearly $5-t_{p}$ are ignorable, so the isotropy group will have dimension $s=\operatorname{dim}\left(H_{p}\right)$, and the group of isometries of the metric will have dimension $r$ given by(see Cartan 43])

$$
r=s+5-t_{p}
$$

acting on an orbit with dimension

$$
d=r-s=5-t_{p}
$$

The line element of the five-dimensional Riemannian Gödel-type manifolds $M_{5}$ we are concerned with in this work is given by

$$
d s^{2}=d t^{2}+2 H(x) d t d y-d x^{2}-G(x) d y^{2}-d z^{2}-d u^{2},
$$

where $H(x)$ and $G(x)$ are arbitrary real functions of $x$, and the five STM coordinates clearly are $t, x, y, z, u$. As a matter of fact, to ensure the local Lorentzian character of (2.6) one has to require that $H^{2}(x)+G(x)>0$. At an arbitrary point of $M_{5}$ one can choose the following set of linearly independent one-forms $\theta^{A}$ :

$$
\theta^{0}=d t+H(x) d y, \quad \theta^{1}=d x, \quad \theta^{2}=D(x) d y, \quad \theta^{3}=d z, \quad \theta^{4}=d u,
$$

such that the Gödel-type line element (2.6) can be written as

$$
d s^{2}=\eta_{A B} \theta^{A} \theta^{B}=\left(\theta^{0}\right)^{2}-\left(\theta^{1}\right)^{2}-\left(\theta^{2}\right)^{2}-\left(\theta^{3}\right)^{2}-\left(\theta^{4}\right)^{2},
$$


where $D^{2}(x)=G+H^{2}$. Here and in what follows capital letters are pentad indices (or Lorentz frame indices) and run from 0 to 4; they are raised and lowered with Lorentz matrices $\eta^{A B}=\eta_{A B}=\operatorname{diag}(+1,-1,-1,-1,-1)$, respectively.

Using as input the one-forms (2.7) and the Lorentz frame (2.8), the computer algebra package CLASSI gives the following nonvanishing Lorentz frame components $R_{A B C D}$ of the curvature:

$$
\begin{aligned}
& R_{0101}=R_{0202}=-\frac{1}{4}\left(\frac{H^{\prime}}{D}\right)^{2} \\
& R_{0112}=\frac{1}{2}\left(\frac{H^{\prime}}{D}\right)^{\prime} \\
& R_{1212}=\frac{D^{\prime \prime}}{D}-\frac{3}{4}\left(\frac{H^{\prime}}{D}\right)^{2}
\end{aligned}
$$

where the prime denotes derivative with respect to $x$.

For STM homogeneity from eq. (2.5) one must have $t_{q}=0$ for $q=0,1, \cdots p$, that is, the number of functionally independent functions of $x^{\mu}$ in the set $I_{p}$ must be zero. Therefore, from eqs. 2.9) - (2.11) we conclude that for STM homogeneity it is necessary that

$$
\begin{aligned}
& \frac{H^{\prime}}{D}=\text { const } \equiv-2 \omega \\
& \frac{D^{\prime \prime}}{D}=\text { const } \equiv m^{2} .
\end{aligned}
$$

We shall now show that the above necessary conditions are also sufficient for STM local homogeneity. Indeed, under these conditions the nonvanishing frame components of the curvature reduce to

$$
\begin{aligned}
& R_{0101}=R_{0202}=-\omega^{2}, \\
& R_{1212}=m^{2}-3 \omega^{2} .
\end{aligned}
$$


Following Cartan's method for the local equivalence, we next calculate the first covariant derivative of the Riemann tensor. Now one obtains the following non-null covariant derivatives of the curvature:

$$
R_{0112 ; 1}=R_{0212 ; 2}=\omega\left(m^{2}-4 \omega^{2}\right)
$$

As the first covariant derivative of the curvature is algebraically expressible in terms of the Riemann tensor and for a given pair $\left(m^{2}, \omega\right)$ the isotropy group $H_{1}$ is the same as $H_{0}$ (see next paragraph), no new covariant derivative of the curvature should be calculated. Clearly one also has $t_{0}=t_{1}=0$.

As far as the dimension of the residual isotropy group is concerned we distinguish two different classes of locally homogeneous 5D Gödel-type Riemannian manifolds, according to the relevant parameters $m^{2}$ and $\omega$, namely [52]

(i) $m^{2} \neq 4 \omega^{2}$ and $\omega \neq 0$ for which $\operatorname{dim}\left(H_{0}\right)=\operatorname{dim}\left(H_{1}\right)=2$;

(ii) $m^{2}=4 \omega^{2}$ with $\omega \neq 0$, and the degenerated Gödel-type manifolds $m^{2} \neq 0$ and $\omega=0$. Here one has $\operatorname{dim}\left(H_{0}\right)=\operatorname{dim}\left(H_{1}\right)=4$.

Thus, from eqs. (2.4) and (2.5) one finds that the locally homogeneous 5D Riemannian Gödel-type manifolds admit a (local) $G_{r}$, with either $r=7$ or $r=9$, acting on an orbit of dimension 5, that is on the whole STM manifold.

The above results can be collected together in the following theorems:

Theorem 1 The necessary and sufficient conditions for a five-dimensional Riemannian Gödel-type manifold to be locally homogeneous are those given by equations (2.19) - (2.13).

Theorem 2 The five-dimensional locally homogeneous Riemannian Gödel-type manifolds admit group of isometry $G_{r}$ with

(i) $r=7$ if $m^{2} \neq 4 \omega^{2}$ and $\omega \neq 0$; 
(ii) $r=9$ if $m^{2}=4 \omega^{2}$ with $\omega \neq 0$, or when $m^{2} \neq 0$ and $\omega=0$.

Theorem 3 The five-dimensional homogeneous Riemannian Gödel-type manifolds are locally characterized by two independent parameters $m^{2}$ and $\omega$ : identical pairs $\left(m^{2}, \omega\right)$ specify locally equivalent manifolds.

We remark that the particular case $m^{2}=\omega=0$ has not been included in our study inasmach as, from (2.14) and (2.15), it is clearly the 5D flat manifold.

We shall now be concerned with the irreducible set of isometrically nonequivalent homogeneous Gödel-type metrics. To this end, we distinguish four classes of metrics according to:

Class I : $m^{2}>0, \omega \neq 0$. For this case, the general solution of $(2.12)$ and $(2.13)$ can be written as

$$
D(x)=a_{0} e^{m x}+a_{1} e^{-m x} \quad \text { and } \quad H(x)=-\frac{2 \omega}{m}\left(a_{0} e^{m x}-a_{1} e^{-m x}\right)+a_{2},
$$

where $a_{0}, a_{1}$ and $a_{2}$ are arbitrary constants. According to the above theorem (3), the constants $a_{0}, a_{1}$ and $a_{2}$ are not essential. In other words they can be eliminated by coordinate transformations. Indeed, if one performs the coordinate transformation $u^{\prime}=u$ and successively the transformations (3.10), (3.15) and (3.16) of [37] one finds that the line element for this class of homogeneous Gödel-type manifolds is brought into the form

$$
d s^{2}=[d t+H(r) d \phi]^{2}-D^{2}(r) d \phi^{2}-d r^{2}-d z^{2}-d u^{2}
$$

in cylindrical coordinates $(r, \phi, z)$, where

$$
H(r)=\frac{2 \omega}{m^{2}}[1-\cosh (m r)] \quad \text { and } \quad D(r)=\frac{1}{m} \sinh (m r) .
$$

Class II : $m^{2}=0, \omega \neq 0$. For this case, the general solution of (2.12) and (2.13) is

$$
D(x)=b_{0} x-b_{1} \quad \text { and } \quad H(x)=-\omega x\left(b_{0} x-2 b_{1}\right)+b_{2},
$$


where $b_{0}, b_{1}$ and $b_{2}$ are arbitrary constants. By trivial coordinate transformations the line element for this class can be brought to the form (2.18) but now with

$$
H(r)=-\omega r^{2} \quad \text { and } \quad D(r)=r,
$$

where only the essential parameter $\omega$ appears $\left(m^{2}=0\right.$, for this class).

Class III : $m^{2} \equiv-\mu^{2}<0, \omega \neq 0$. Similarly for this class, the integration of the conditions for homogeneity $(2.12)$ and $(2.13)$ leads to

$$
D(x)=c_{0} \sin (\mu x)+c_{1} \cos (\mu x) \text { and } \quad H(x)=\frac{2 \omega}{\mu}\left[c_{0} \cos (\mu x)-c_{1} \sin (\mu x)\right]+c_{2} .
$$

Here again the non-essential constants $c_{0}, c_{1}$ and $c_{2}$ can be eliminated by coordinate transformations so that the line element for this class reduces to (2.18) with

$$
H(r)=\frac{2 \omega}{\mu^{2}}[\cos (\mu r)-1] \quad \text { and } \quad D(r)=\frac{1}{\mu} \sin (\mu r)
$$

Class IV : $m^{2} \neq 0, \omega=0$. We refer to the manifolds of this class as degenerated Gödel-type manifolds, since the cross term in the line element, related to the rotation $\omega$ in Gödel model, vanishes. By a trivial coordinate transformation one can make $H=0$ with $D(r)$ given, respectively, by (2.19) or (2.23) depending on whether $m^{2}>0$ or $m^{2} \equiv$ $-\mu^{2}<0$

In three out of the above four classes of homogeneous Gödel-type manifolds there are closed timelike curves. Indeed, the Gödel's analysis for 4D manifolds can be extended to the 5D manifolds in a straightfoward way to prove this. To this end, we write the line element (2.18) as

$$
d s^{2}=d t^{2}+2 H(r) d t d \phi-d r^{2}-G(r) d \phi^{2}-d z^{2}-d u^{2},
$$

where $G(r)=D^{2}-H^{2}$ and $(r, \theta, \phi)$ are cylindrical coordinates. 
The existence of closed timelike curves of the Gödel-type depends on the behavior of $G(r)$. Indeed, if $G(r)<0$ for a certain range of $r\left(r_{1}<r<r_{2}\right.$, say), Gödel's circles [53] $u, t, z, r=$ const are closed timelike curves.

It is, therefore, easy to show that for the above class II there is a critical radius $r_{c}$, defined by $|\omega| r_{c}=1$, such that for $r_{c}<r<\infty$ the circles $u, t, z=$ const and $r=$ const $>r_{c}$ are closed timelike curves. So there is breakdown of causality for this class.

For the class III noncausal Gödel circles occur depending on the root $r_{n}$ of the equation

$$
\sin ^{2}\left[\frac{\mu r_{n}}{2}\right]=\left[1+\frac{4 \omega^{2}}{\mu^{2}}\right]^{-1} \leq 1 .
$$

Thus, for this class there are alternatively causal and noncausal regions (circular rings) in the surfaces $u, t, z=$ const.

For the class I if $m^{2}<4 \omega^{2}$ the noncausal Gödel circles occur for $r>r_{1}$, where

$$
\sinh ^{2}\left[\frac{m r_{1}}{2}\right]=\left[1+\frac{4 \omega^{2}}{m^{2}}\right]^{-1} \geq 1 .
$$

However, for $m^{2} \geq 4 \omega^{2}$ then $G(r)>0$ and there is no breakdown of causaly of Gödel-type. As a matter of fact, following Calvão's reasoning [53] one can show that: $(a)$ although causal, the $m^{2}=4 \omega^{2}$ manifolds are not stably causal [50], that is, an arbitrary small perturbation of this metric gives rise to causality violation; $(b)$ the family $m^{2}>4 \omega^{2}$ is stably causal, which means that the causal properties of this family are invariant under arbitrary small perturbation of its metrics. We remark that Calvão's approach holds only if the 5D homogeneous Riemannian Gödel-type manifolds are endowed with the topology of $\mathcal{R}^{5}$.

Finally, for the degenerated Gödel-type geometries (class IV), as $G(r)=D^{2}>0$ there is no closed timelike curves of Gödel type. 


\section{Isometries}

In this section we shall be concerned with the isometries of the homogeneous $5 \mathrm{D}$ Riemannian Gödel-type manifolds, whose line element (2.18) can be brought into the Lorentzian form (2.8) but now with the one-forms $\theta^{A}$ given by

$$
\theta^{0}=d t+H(r) d \phi, \quad \theta^{1}=d r, \quad \theta^{2}=D(r) d \phi, \quad \theta^{3}=d z, \quad \theta^{4}=d u
$$

where the functions $H(r)$ and $D(r)$ are given by (2.19), (2.21) or (2.23) depending on the sign of $m^{2}$.

Denoting the coordinate components of a generic Killing vector field $K$ by $K^{\mu} \equiv$ $(Q, R, S, Z, U)$, where $Q, R, S, Z$ and $U$ are functions of all coordinates $t, r, \phi, z, u$, then the fifteen Killing equations

$$
K_{(A ; B)} \equiv K_{A ; B}+K_{B ; A}=0
$$

can be written in the Lorentz frame (2.8) - 3.1) as

$$
\begin{gathered}
T_{t}=0, \quad T_{z}-Z_{t}=0, \\
R_{r}=0, \quad Z_{r}+R_{z}=0, \\
Z_{z}=0, \\
D\left(T_{r}-R_{t}\right)-H_{r} P=0, \\
D P_{z}+Z_{\phi}-H Z_{t}=0, \\
T_{\phi}+H_{r} R-D P_{t}=0, \\
R_{\phi}-H R_{t}-D_{r} P+D P_{r}=0, \\
P_{\phi}-H P_{t}+D_{r} R=0, \\
T_{u}-U_{t}=0
\end{gathered}
$$




$$
\begin{gathered}
R_{u}+U_{r}=0, \\
D P_{u}-H U_{t}+U_{\phi}=0, \\
U_{z}+Z_{u}=0, \\
U_{u}=0
\end{gathered}
$$

where the subscripts denote partial derivatives, and where we have made

$$
T \equiv H S+Q \quad \text { and } \quad P \equiv D S
$$

to make easier the comparison and the use of the results obtained in [38]. To this end we note that in the present form the equations $(3.3)-(3.10)$ are formally identical to the Killing equations (4) to (11) of [38]. However, in the above equations $(3.3)-(3.10)$ the functions $T, R, P, Z$ depend additionally on the fifth coordinate $u$. Taking into account this similitude, the integration of the Killing equations (3.3) - (3.15) can be obtained in two steps as follows. First, by analogy with (4) to (11) of [38 one integrates (3.3) - (3.10), but at this step instead of the integration constants one has integration functions of the fifth coordinate $u$. Second, one uses the remaining eqs. (3.11) - (3.15) to achieve explicit forms for these integration functions and to obtain the last component $U$ of $K$.

In what follows we shall use the above two-steps procedure to integrate the Killing equations (3.3) - (3.15) for the class I of the section 2 in details. However, for the sake of brevity, for the remaining classes we shall only present the Killing vector fields and the corresponding Lie algebras without going into details of calculation, which can be similarly obtained, or simply verified by using the computer algebra program KILLNF, written in CLASSI by Åman [41], with further extensions by MacCallum [42]. As a matter of fact, even the Killing equations (3.3) - (3.15) have been achieved by using KILLNF.

Class I : $m^{2}>0, \omega \neq 0$. In the integration of the Killing equation for this general class 
one is led to distinguish two different classes of solutions depending on whether $m^{2} \neq 4 \omega^{2}$ or $m^{2}=4 \omega^{2}$. We shall refer to these subclasses as classes Ia and Ib, respectively.

Class Ia : $m^{2}>0, m^{2} \neq 4 \omega^{2}$. According to the above two-steps procedure, from (3.16) and according to eqs. (36) - (39) of 38] one finds

$$
\begin{aligned}
Q= & \alpha+2 \omega\left(\gamma D_{r}+\delta D \sin \phi+\epsilon D \cos \phi\right) \\
& +\frac{H}{D}\left(\gamma m^{2} D+\delta D_{r} \sin \phi+\epsilon D_{r} \cos \phi\right), \\
R= & \delta \cos \phi-\epsilon \sin \phi, \\
S= & -\gamma m^{2}-\frac{D_{r}}{D}(\delta \sin \phi+\epsilon \cos \phi), \\
Z= & \beta, \\
U= & U(t, r, \phi, z, u),
\end{aligned}
$$

where $\alpha, \beta, \gamma, \delta$ and $\epsilon$ are integration functions of the fifth coordinate $u$.

From (3.14) and (3.15) one finds

$$
\begin{aligned}
\beta & =\kappa_{7} u+\kappa_{2}, \\
U & =-\kappa_{7} z+\zeta(t, r, \phi),
\end{aligned}
$$

where $\kappa_{2}$ and $\kappa_{7}$ are arbitrary constants and $\zeta(t, r, \phi)$ is an arbitrary function.

Equation (3.12) can now be used to obtain the integration functions $\delta$ and $\epsilon$ and to simplify the expression of $U$. In doing so one finds

$$
\begin{gathered}
\delta=\delta_{0} u+\kappa_{4}, \quad \epsilon=\epsilon_{0} u+\kappa_{5}, \\
U=-\kappa_{7} z-\delta_{0} r \cos \phi+\epsilon_{0} r \sin \phi+\nu(t, \phi),
\end{gathered}
$$

where $\delta_{0}, \epsilon_{0}, \kappa_{4}, \kappa_{5}$ are constants and $\nu(t, \phi)$ is an arbitrary function. 
Finally, equations (3.11) and (3.12) give the remaining integration functions $\alpha, \gamma$ and reduce the functions $\delta, \epsilon$ and $U$ to

$$
\begin{aligned}
& \gamma=\text { const } \equiv m^{-1} \kappa_{3}, \quad \alpha=\text { const } \equiv \kappa_{1}, \\
& \delta=\kappa_{4}, \quad \epsilon=\kappa_{5}, \quad U=-\kappa_{7} z+\kappa_{6},
\end{aligned}
$$

where $\kappa_{6}$ is a new arbitrary constant.

Inserting (3.22) and (3.26) - (3.27) into (3.17) - (3.21) and using (2.19) one obtains

$$
\begin{aligned}
Q & =\kappa_{1}+\frac{2 \omega}{m} \kappa_{3}-\frac{H}{D}\left(\kappa_{4} \sin \phi+\kappa_{5} \cos \phi\right) \\
R & =\kappa_{4} \cos \phi-\kappa_{5} \sin \phi \\
S & =-\kappa_{3} m-\frac{D_{r}}{D}\left(\kappa_{4} \sin \phi+\kappa_{5} \cos \phi\right), \\
Z & =\kappa_{7} u+\kappa_{2} \\
U & =-\kappa_{7} z+\kappa_{6} .
\end{aligned}
$$

Thus, in the coordinate basis in which as (2.18) is given, a set of linearly independent Killing vector fields $K_{N}$ ( $N$ is an enumerating index) can be written as

$$
\begin{aligned}
& K_{1}=\partial_{t}, \quad K_{2}=\partial_{z}, \quad K_{3}=\frac{2 \omega}{m} \partial_{t}-m \partial_{\phi}, \\
& K_{4}=-\frac{H}{D} \sin \phi \partial_{t}+\cos \phi \partial_{r}-\frac{D_{r}}{D} \sin \phi \partial_{\phi}, \\
& K_{5}=-\frac{H}{D} \cos \phi \partial_{t}-\sin \phi \partial_{r}-\frac{D_{r}}{D} \cos \phi \partial_{\phi}, \\
& K_{6}=\partial_{u}, \quad K_{7}=u \partial_{z}-z \partial_{u} .
\end{aligned}
$$

The Lie algebra has the following nonvanishing commutators:

$$
\begin{gathered}
{\left[K_{2}, K_{7}\right]=-K_{6}, \quad\left[K_{3}, K_{4}\right]=-m K_{5},} \\
{\left[K_{3}, K_{5}\right]=m K_{4}, \quad\left[K_{4}, K_{5}\right]=m K_{3},} \\
{\left[K_{6}, K_{7}\right]=K_{2} .}
\end{gathered}
$$


So, the corresponding algebra is $\mathcal{L}_{I a}=t^{2} \oplus s o(2) \oplus \tau \oplus s o(2,1)$. The semi-direct sum of sub-algebras $t^{2} \in s o(2)$ corresponds to the translations $K_{2}$ and $K_{6}$, and the rotation $K_{7}$. The symbol $\tau$ is associated to the time translation $K_{1}$. Finally, the infinitesimal generators of sub-algebra so $(2,1)$ are $K_{3}, K_{4}$ and $K_{5}$.

Class Ib : $m^{2}=4 \omega^{2}, \omega \neq 0$. For this class, using the results of [38] and (3.16) one finds

$$
\begin{aligned}
Q & =\alpha+\gamma-\frac{H}{D}(\delta \sin \phi+\epsilon \cos \phi)-\frac{H}{D}[\eta \sin (m t+\phi)+\xi \cos (m t+\phi)], \\
R & =\delta \cos \phi-\epsilon \sin \phi+\xi \sin (m t+\phi)-\eta \cos (m t+\phi), \\
S & =-\gamma m-\frac{D_{r}}{D}(\delta \sin \phi+\epsilon \cos \phi)+\frac{1}{D}[\eta \sin (m t+\phi)+\xi \cos (m t+\phi)], \\
Z & =Z(u), \\
U & =U(t, r, \phi, z, u),
\end{aligned}
$$

where $\alpha, \gamma, \delta, \epsilon, \xi$ and $\eta$ are (new) integration functions of the fifth coordinate $u$.

Again, from (3.14) and (3.15) one finds

$$
\begin{aligned}
& Z=k_{8} u+k_{2}, \\
& U=-k_{8} z+\chi(t, r, \phi),
\end{aligned}
$$

where $k_{2}$ and $k_{8}$ are arbitrary constants and $\chi(t, r, \phi)$ is an arbitrary function.

Similarly to the class Ia, eqs. (3.12) can be used to obtain a first expression for the integration functions $\xi, \eta \delta, \epsilon$, and to reduce $U$ according to

$$
\begin{gathered}
\xi=\xi_{0} u+k_{6}, \quad \eta=\eta_{0} u+k_{7}, \\
\delta=\delta_{0} u+k_{4}, \quad \epsilon=\epsilon_{0} u+k_{5}, \\
U=-k_{8} z+r\left[-\delta_{0} \cos \phi+\epsilon_{0} \sin \phi-\xi_{0} \sin (m t+\phi)+\eta_{0} \cos (m t+\phi)\right]+\sigma(t, \phi),
\end{gathered}
$$


where $\xi_{0}, \eta_{0}, \delta_{0}, \epsilon_{0}, k_{4}, \cdots, k_{7}$ are constants, and $\sigma(t, \phi)$ is an arbitrary function.

Equations (3.11) and (3.12) give the remaining integration functions $\alpha, \gamma$ and reduce the functions $\xi, \eta, \delta, \epsilon$ and $U$ to

$$
\begin{aligned}
\xi & =k_{6}, \quad \eta=k_{7}, \quad \gamma=\text { const } \equiv k_{3}, \quad \alpha=\text { const } \equiv k_{1}, \\
\delta & =k_{4}, \quad \epsilon=k_{5}, \quad U=-k_{8} z+k_{9},
\end{aligned}
$$

where $k_{9}$ is a new arbitrary constant.

Inserting $(3.45)$ and $(3.50)-(3.51)$ into 3.40$)-(3.44)$ one obtains

$$
\begin{aligned}
& Q=k_{1}+k_{3}-\frac{H}{D}\left(k_{4} \sin \phi+k_{5} \cos \phi\right)-\frac{H}{D}\left[k_{7} \sin (m t+\phi)+k_{6} \cos (m t+\phi)\right], \\
& R=k_{4} \cos \phi-k_{5} \sin \phi+k_{6} \sin (m t+\phi)-k_{7} \cos (m t+\phi) \\
& S=-k_{3} m-\frac{D_{r}}{D}\left(k_{4} \sin \phi+k_{5} \cos \phi\right)+\frac{1}{D}\left[k_{7} \sin (m t+\phi)+k_{6} \cos (m t+\phi)\right], \\
& Z=k_{8} u+k_{2} \\
& U=-k_{8} z+k_{9}
\end{aligned}
$$

which give rise to the Killing vector fields

$$
\begin{aligned}
K_{1} & =\partial_{t}, \quad K_{2}=\partial_{z}, \quad K_{3}=\partial_{t}-m \partial_{\phi}, \\
K_{4} & =-\frac{H}{D} \sin \phi \partial_{t}+\cos \phi \partial_{r}-\frac{D_{r}}{D} \sin \phi \partial_{\phi}, \\
K_{5} & =-\frac{H}{D} \cos \phi \partial_{t}-\sin \phi \partial_{r}-\frac{D_{r}}{D} \cos \phi \partial_{\phi}, \\
K_{6} & =-\frac{H}{D} \cos (m t+\phi) \partial_{t}+\sin (m t+\phi) \partial_{r}+\frac{1}{D} \cos (m t+\phi) \partial_{\phi}, \\
K_{7} & =-\frac{H}{D} \sin (m t+\phi) \partial_{t}-\cos (m t+\phi) \partial_{r}+\frac{1}{D} \sin (m t+\phi) \partial_{\phi}, \\
K_{8} & =u \partial_{z}-z \partial_{u}, \quad K_{9}=\partial_{u},
\end{aligned}
$$

whose Lie algebra is given by

$$
\left[K_{1}, K_{6}\right]=-m K_{7}, \quad\left[K_{1}, K_{7}\right]=m K_{6}, \quad\left[K_{2}, K_{8}\right]=-K_{9},
$$




$$
\begin{gathered}
{\left[K_{3}, K_{4}\right]=-m K_{5}, \quad\left[K_{3}, K_{5}\right]=m K_{4}, \quad\left[K_{4}, K_{5}\right]=m K_{3},} \\
{\left[K_{6}, K_{7}\right]=m K_{1}, \quad\left[K_{8}, K_{9}\right]=-K_{2} .}
\end{gathered}
$$

So, the corresponding algebra for this case is $\mathcal{L}_{I b}=t^{2} \oplus s o(2) \oplus s o(2,1) \oplus s o(2,1)$. The semi-direct sum $t^{2} \in s o(2)$ comprises the translations $K_{2}$ and $K_{9}$, and the rotation $K_{8}$, while the two sub-algebras so $(2,1)$ are generated by the Killing vector fields $K_{1}, K_{6}, K_{7}$ and $K_{3}, K_{4}, K_{5}$.

Class II : $m^{2}=0, \omega \neq 0$. Similarly for this class, using 2.21 and the results of 37 the above two-steps procedure gives rise to the following Killing vector fields:

$$
\begin{aligned}
& K_{1}=\partial_{t}, \quad K_{2}=\partial_{z}, \quad K_{3}=\partial_{\phi}, \\
& K_{4}=-\omega r \sin \phi \partial_{t}-\cos \phi \partial_{r}+\frac{1}{r} \sin \phi \partial_{\phi}, \\
& K_{5}=-\omega r \cos \phi \partial_{t}+\sin \phi \partial_{r}+\frac{1}{r} \cos \phi \partial_{\phi}, \\
& K_{6}=u \partial_{z}-z \partial_{u}, \quad K_{7}=\partial_{u} .
\end{aligned}
$$

The Lie algebra has the following nonvanishing commutators:

$$
\begin{gathered}
{\left[K_{2}, K_{6}\right]=-K_{7}, \quad\left[K_{3}, K_{4}\right]=K_{5},} \\
{\left[K_{3}, K_{5}\right]=-K_{4}, \quad\left[K_{4}, K_{5}\right]=2 \omega K_{1},} \\
{\left[K_{6}, K_{7}\right]=-K_{2} .}
\end{gathered}
$$

Therefore, the corresponding algebra for this case is $\mathcal{L}_{I I}=t^{2} \in s o(2) \oplus \mathcal{L}_{4}$. The semidirect sum of sub-algebras corresponds here to the translations $K_{2}$ and $K_{7}$, and the rotation $K_{6}$. The sub-algebra $\mathcal{L}_{4}$ is generated by $K_{1}, K_{3}, K_{4}$ and $K_{5}$. This algebra $\mathcal{L}_{4}$ is soluble and does not contain abelian 3D sub-algebras; it is classified as type $I I I$ with $q=0$ by Petrov [54]. 
Class III : $m^{2} \equiv-\mu^{2}<0, \omega \neq 0$. Again for this class using (2.23) and [38, the above outlined two-step procedure furnishes

$$
\begin{aligned}
& K_{1}=\partial_{t}, \quad K_{2}=\partial_{z}, \quad K_{3}=\frac{2 \omega}{\mu} \partial_{t}+\mu \partial_{\phi}, \\
& K_{4}=-\frac{H}{D} \sin \phi \partial_{t}+\cos \phi \partial_{r}-\frac{D_{r}}{D} \sin \phi \partial_{\phi}, \\
& K_{5}=-\frac{H}{D} \cos \phi \partial_{t}-\sin \phi \partial_{r}-\frac{D_{r}}{D} \cos \phi \partial_{\phi}, \\
& K_{6}=u \partial_{z}-z \partial_{u}, \quad K_{7}=\partial_{u} .
\end{aligned}
$$

The Lie algebra has the following nonvanishing commutators:

$$
\begin{gathered}
{\left[K_{2}, K_{6}\right]=-K_{7}, \quad\left[K_{3}, K_{4}\right]=\mu K_{5},} \\
{\left[K_{3}, K_{5}\right]=-\mu K_{4}, \quad\left[K_{4}, K_{5}\right]=\mu K_{3},} \\
{\left[K_{6}, K_{7}\right]=-K_{2} .}
\end{gathered}
$$

Thus, the corresponding algebra for this case is $\mathcal{L}_{I I I}=t^{2} \in s o(2) \oplus \tau \oplus$ so (3). The semi-direct sum of sub-algebras corresponds again to the translations $K_{2}$ and $K_{7}$, and the rotation $K_{6}$. Here $\tau$ is associated to the Killing vector field $K_{1}$, whereas to the sub-algebra so (3) correspond $K_{3}, K_{4}$ and $K_{5}$.

Class IV : $m^{2} \neq 0, \omega=0$. This class corresponds to the so-called degenerated Gödeltype manifolds. By a similar procedure one obtains for this class the following Killing vector fields:

$$
\begin{aligned}
& K_{1}=\partial_{t}, \quad K_{2}=\partial_{z}, \quad K_{3}=z \partial_{t}+t \partial_{z}, \\
& K_{4}=\cos \phi \partial_{r}-\frac{D_{r}}{D} \sin \phi \partial_{\phi}, \\
& K_{5}=-\sin \phi \partial_{r}-\frac{D_{r}}{D} \cos \phi \partial_{\phi}, \quad K_{6}=\partial_{\phi}, \\
& K_{7}=u \partial_{z}-z \partial_{u}, \quad K_{8}=u \partial_{t}+t \partial_{u}, \quad K_{9}=\partial_{u},
\end{aligned}
$$


where $D(r)=(1 / m) \sinh m r$ for $m^{2}>0$, or $D(r)=(1 / \mu) \sin \mu r$ for $m^{2} \equiv-\mu^{2}<0$. The Lie algebra has the following nonvanishing commutators:

$$
\begin{aligned}
& {\left[K_{1}, K_{3}\right]=K_{2}, \quad\left[K_{1}, K_{8}\right]=K_{9}, \quad\left[K_{2}, K_{3}\right]=K_{1},} \\
& {\left[K_{2}, K_{7}\right]=-K_{9}, \quad\left[K_{3}, K_{7}\right]=-K_{8}, \quad\left[K_{3}, K_{8}\right]=-K_{7},} \\
& {\left[K_{4}, K_{5}\right]=-m^{2} K_{6}, \quad\left[K_{4}, K_{6}\right]=-K_{5}, \quad\left[K_{5}, K_{6}\right]=K_{4},} \\
& {\left[K_{7}, K_{8}\right]=-K_{3}, \quad\left[K_{7}, K_{9}\right]=-K_{2}, \quad\left[K_{8}, K_{9}\right]=-K_{1},}
\end{aligned}
$$

where one should substitute $-m^{2}$ by $\mu^{2}$ if $m^{2}<0$. So, when $m^{2}>0$ the corresponding algebra is $\mathcal{L}_{I V}=t^{3} \in s o(2,1) \oplus s o(2,1)$, where to $t^{3}$ correspond $K_{1}, K_{2}$ and $K_{9}$. The infinitesimal generators of $s o(2,1)$ in the semi-direct sum are $K_{3}, K_{7}$ and $K_{8}$, while to the other so $(2,1)$ correspond $K_{4}, K_{5}$ and $K_{6}$. When $m^{2}<0$ the algebra associated to $K_{4}, K_{5}$ and $K_{6}$ is $s o(3)$, instead.

It is worth noting that none of the above Lie algebras is semi-simple, but some of their sub-algebras are. Besides, most of the simple sub-algebras are non-compact. The 3D sub-algebra so (3) present in the cases $\mathcal{L}_{I I I}$ and $\mathcal{L}_{I V}$ with $m^{2}<0$ are compact, though.

We have therefore succeeded in finding the maximal group of motions of all classes of 5D homogeneous Riemannian Gödel-type manifolds we have studied in the previous section.

Equations $(3.33)-(3.36),(3.57)-(3.62),(3.66)-(3.69),(3.73)-(3.76)$, and $(3.80)-$ (3.83) make explicit that the 5D locally homogeneous Riemannian Gödel-type manifolds admit maximal group of isometry $G_{r}$ with: $(a) r=7$ if $m^{2} \neq 4 \omega^{2}$ and $\omega \neq 0$; or $(b)$ $r=9$ if $m^{2}=4 \omega^{2}$ with $\omega \neq 0$, or when $m^{2} \neq 0$ and $\omega=0$, in agreement with theorem 2 of the previous section. Actually the integration of the Killing equations constitutes a different way of deriving that theorem. Furthermore, these equations also show that the 
isotropy subgroup $H$ of $G_{r}$ is such that $\operatorname{dim}(H)=2$ for $m^{2} \neq 4 \omega^{2}$ and $\omega \neq 0$, while for $m^{2}=4 \omega^{2}$ with $\omega \neq 0$ as well as for the degenerated Gödel-type $\left(m^{2} \neq 0\right.$ and $\left.\omega=0\right)$ we have $\operatorname{dim}(H)=4$, also in agreement with the previous section.

To conclude, we should like to emphasize again that the results of this work hold for any five-dimensional locally homogeneous Riemannian Gödel-type manifold, regardless of the underlying 5D Kaluza-Klein-type theory of gravitation one may be concerned with. This gives a measure of the generality of our results in the context of these theories, in which the 5D Riemannian manifolds are the underlying arena for the formulation of the physical laws.

\section{Acknowledgement}

The authors gratefully acknowledge financial assistance from CNPq. 


\section{References}

[1] G. Nordström, Phys. Z. 15, 504 (1914).

[2] Th. Kaluza, Sitzungsberichte preuss. Akad. Wiss. - Phys. Math. Klasse LIV, 966 (1921).

[3] O. Z. Klein, Phys. Z. 37, 895 (1926).

[4] A. Chodos and S. Detweiler, Phys. Rev. D 21, 2167 (1980).

[5] E. Alvarez, Phys. Rev. Lett. 51, 931 (1983).

[6] A. H. Guth, Phys. Rev. D 23, 347 (1981).

[7] L. Burakovsky and L.P. Horwitz, Gen. Rel. Grav. 27, 1043 (1995).

[8] J. Ibáñez and E. Verdaguer, Phys. Rev. D 34, 1202 (1986).

[9] R.J. Gleiser and M.C. Diaz, Phys. Rev. D 37, 3761 (1986). See also references therein.

[10] Y. S. Vladimirov and S. S. Kokarev, Gen. Rel. Grav. 29, 141 (1997).

[11] P. S. Wesson, Astron. Astrophys. 119, 145 (1983).

[12] P. S. Wesson, Gen. Rel. Grav. 16, 193 (1984).

[13] P. S. Wesson, Astron. Astrophys. 143, 233 (1985).

[14] Ø. Grøn and H. H. Soleng, Gen. Rel. Grav. 20, 1115 (1988) .

[15] P. S. Wesson, Gen. Rel. Grav. 22, 707 (1990).

[16] G.-W. Ma, Phys. Lett. A 146, 375 (1990). 
[17] Ø. Grøn, Astron. Astrophys. 193, 1 (1988).

[18] P. S. Wesson, Astron. Astrophys. 166, 1 (1986).

[19] A. Coley, Astron. Astrophys. 233, 305 (1990).

[20] K. Gödel, Rev. Mod. Phys. 21, 447 (1949).

[21] M. M. Som and A. K. Raychaudhuri, Proc. Roy. Soc. London A 304, 81 (1968).

[22] A. Banerjee and S. Banerji, J. Phys. A 1, 188 (1968).

[23] F. Bampi and C. Zordan, Gen. Rel. Grav. 9, 393 (1978).

[24] M. Novello and M. J. Rebouças, Phys. Rev. D 19, 2850 (1979).

[25] M. J. Rebouças, Phys. Lett. A 70, 161 (1979).

[26] J. Pfarr, Gen. Rel. Grav. 13, 1073 (1981). See also references therein quoted on the philosophical discussions which came about after Gödel's paper.

[27] S. K. Chakraborty, Gen. Rel. Grav. 12, 925 (1980).

[28] M. J. Rebouças and A. F. F. Teixeira, Phys. Rev. D 34, 2985 (1986).

[29] M. J. Rebouças, J. E. Åman and A. F. F. Teixeira, J. Math. Phys. 27, 1370 (1986).

[30] F. M. Paiva, M. J. Rebouças and A. F. F. Teixeira, Phys. Lett. A 126, 168 (1987).

[31] K. Dunn, Gen. Rel. Grav. 21, 137 (1989).

[32] R. X. Saibatalov, Gen. Rel. Grav. 7, 697 (1995). 
[33] T. Singh and A. K. Agrawal, Fortschr. Phys. 42, 71 (1994). It contains a fair list of references on Gödel-type space-times in general relativity and in the torsion theories of gravitation.

[34] A. Krasiński, Rotating Dust Solutions of Einstein's Equations with 3-Dimensional Symmetry Groups. Part 1: All Killing Fields Linearly Independent of $u^{\alpha}$ and $\omega^{\alpha}$, grqc/9607021 (1997). This reference contains an overview of the literature on rotating models in general relativity.

[35] For a clear distinction between local and global (topological) homogeneity of a manifold see, for example, T. Koike, M. Tanimoto and A. Hosoya, J. Math. Phys. 35, 4855 (1994).

[36] A. K. Raychaudhuri and S. N. G. Thakurta, Phys. Rev. D 22, 802 (1980).

[37] M. J. Rebouças and J. Tiomno, Phys. Rev. D 28, 1251 (1983).

[38] A. F. F. Teixeira, M. J. Rebouças and J. E. Åman, Phys. Rev. D 32, 3309 (1985). Note that in this reference there are two misprints, namely: in eq.(5) the term $-R_{z}$ should read $+R_{z}$, and in eq. (38) the term $\kappa_{3} m D$ should read $\kappa_{3} m^{2} D$.

[39] M. J. Rebouças and J. E. Åman, J. Math. Phys. 28, 888 (1987).

[40] A. Karlhede, Gen. Rel. Grav. 12, 693 (1980).

[41] J. E. Åman, "Manual for CLAssi: Classification Programs for Geometries in General Relativity", Institute of Theoretical Physics Technical Report, 1987. Third provisional edition. Distributed with the SHEEP sources. 
[42] M. A. H. MacCallum and J. E. F. Skea, "SheEP: A Computer Algebra System for General Relativity", in Algebraic Computing in General Relativity, Lecture Notes from the First Brazilian School on Computer Algebra, Vol. II, edited by M. J. Rebouças and W. L. Roque. Oxford U. P., Oxford (1994). See also references therein.

[43] E. Cartan, "Leçons sur la Géométrie des Éspaces de Riemann", Gauthier-Villars, Paris (1951). English translation by J. Glazebrook, Math. Sci. Press, Brookline (1983).

[44] P. S. Wesson, Astrophys. J. 394, 19 (1992).

[45] P. S. Wesson, Phys. Lett. B 276, 299 (1992).

[46] P. S. Wesson and J. Ponce de Leon, J. Math. Phys. 33, 3883 (1992).

[47] A. Billyard and P. S. Wesson, Gen. Rel. Grav. 28, 129 (1997). See also the references therein cited on a new 5D Kaluza-Klein-type theory of gravitation.

[48] M. A. H. MacCallum, "Classifying Metrics in Theory and Practice", in Unified Field Theory in More Than 4 Dimensions, Including Exact Solutions, edited by V. de Sabbata and E. Schmutzer. World Scientific, Singapore (1983).

[49] M. A. H. MacCallum, "Computer-aided Classification of Exact Solutions in General Relativity", in General Relativity and Gravitational Physics (9th Italian Conference), edited by R. Cianci, R. de Ritis, M. Francaviglia, G. Marmo, C. Rubano and P. Scudellaro. World Scientific, Singapore (1991).

[50] S. W. Hawking and G. F. R. Ellis, "The Large Scale Structure of Space-Time", Cambridge U. P., Cambridge (1973). 
[51] T. Y. Thomas, "Differential Invariants of Generalized Spaces", Cambridge U. P., Cambridge (1934).

[52] In the next section we integrate the Killing equations for the 5D Riemannian Gödeltype manifolds and explicitly show that the isotropy group depends on these special relations between the essential parameters $m^{2}$ and $\omega$.

[53] M. O. Calvão, M. J. Rebouças, A. F. F. Teixeira, W. M. Silva-Jr., J. Math. Phys. 29, 1127 (1988).

[54] A. Z. Petrov, "Einstein Spaces", first English edition, Pergamon Press (1969). See page 63 . 